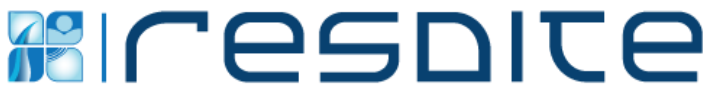

\section{Efetividade da tomografia de impedância elétrica torácica na monitorização de pacientes em ventilação mecânica}

\author{
Effectiveness of thoracic electrical impedance tomography in monitoring patients on \\ mechanical ventilation
}

\section{Roberto Carlos Lyra da Silva ${ }^{1}$, Antônio Augusto de Freitas Peregrino ${ }^{2}$, Daniel Aragão Machado $^{3}$, Carlos Roberto Lyra da Silva ${ }^{4}$, Cristiane Rocha de Oliveira ${ }^{5}$, Cristiano Bertolossi Marta ${ }^{6}$}

\section{Resumo}

Objetivos: avaliar a efetividade da Tomografia de Impedância Elétrica Torácica. Método: Parecer Técnico Científico nos moldes de uma revisão sistemática. As buscas foram feitas no mês de agosto de 2017, nas bases de dados Cochrane Library, MEDLINE (Via Portal PUBMED), BVS, ECRI Institute, PEDRo, EMBASE, Banco de Teses e Dissertações da CAPES e Google Scholar. Resultados: Foram incluídos 14 estudos que mostraram que, muito embora possam existir limitações acerca do uso do TIE para a monitorização pulmonar, sobretudo relacionado à baixa resolução espacial e a necessidade de calibração, a tecnologia parece fornecer informações únicas sobre mecânica pulmonar regional, possibilitando, portanto, que a equipe possa avaliar o suporte ventilatório, otimizando-o para evitar o estresse fisiológico e a tensão do parênquima pulmonar, particularmente em doentes em uso de PEEP. Conclusão: Não foram encontradas evidências sobre a efetividade do TIE na redução do tempo de internação e do tempo de ventilação mecânica invasiva. As evidências foram avaliadas como Grau de Recomendação $B$ e $C$, segundo $o$ Nível de Evidência de Oxford Centre for Evidence Based Medicine) e a recomendação foi fraca a favor da tecnologia.

Palavras chave: Monitoramento. Respiração Artificial. Efetividade

\section{Abstract}

Objectives: To evaluate the effectiveness of thoracic electrical impedance tomography. Method: Scientific Technical Opinion along the lines of a systematic review. Searches were

\footnotetext{
1 PhD, Professor Associado da Universidade Federal do Estado do Rio de Janeiro - UNIRIO. Email: proflyra@gmail.com 2 PhD, Pós-Doutorado. Professor Associado da Universidade do Estado do Rio de Janeiro - UERJ. Email: antonio.peregrino@gmail.com

3 PhD, Professor Adjunto da Universidade Federal do Estado do Rio de Janeiro - UNIRIO. Email: daragao23@gmail.com 4 PhD, Pós-Doutorado, Professor Associado da Universidade Federal do Estado do Rio de Janeiro - UNIRIO. Email: profunirio@gmail.com

5 Msc. Enfermeira do Instituto de Traumatologia e Ortopedia do Estado do Rio de Janeiro - INTO. Email: tiane.hto@gmail.com

6 PhD, Pós-Doutorado, Professor Adjunto da Universidade do Estado do Rio de Janeiro - UERJ. Email: cristianobertol@gmail.com

Correspondência: centro de Ciências Biológicas e da Saúde, Escola de Enfermagem UNIRIO. Rua Xavier Sigaud, Rio de Janeiro, RJ - Brasil. CEP: 22745271.
}

Rev. Saúde Digital Tec. Educ., Fortaleza, CE, v. 5, n.1, p.16-36, jan./abr. 2020. 
performed in August 2017 in the Cochrane Library, MEDLINE (Via PUBMED Portal), VHL, ECRI Institute, PEDRo, EMBASE, CAPES and Google Scholar Theses and Dissertations databases. Results: We included 14 studies that showed that although there may be limitations on the use of EIT for pulmonary monitoring, especially related to low spatial resolution and the need for calibration, the technology seems to provide unique information on regional pulmonary mechanics, enabling, therefore, the team can evaluate ventilatory support, optimizing it to avoid physiological stress and pulmonary parenchymal tension, particularly in patients using PEEP. Conclusion: There was no evidence on the effectiveness of EIT in reducing the length of stay and the time of invasive mechanical ventilation. Evidence was assessed as Degree of Recommendation $B$ and $C$ according to the Oxford Center for Evidence Based Medicine Level of Evidence) and the recommendation was weak in favor of technology.

Keywords: Monitoring. Artificial Respiration. Effectiveness.

\section{Introdução}

Um Parecer Técnico Científico (PTC) visa apresentar os resultados de uma avaliação preliminar que responda, com rapidez, às questões iniciais sobre uma tecnologia, como as evidências de eficácia e segurança ${ }^{1,2,3}$.

Esta proposta foi para atender demanda do Departamento do Complexo Industrial e Inovação em Saúde (DECIIS), área técnica do Ministério da Saúde MS, para resolução dos problemas prioritários de saúde da população brasileira e para o fortalecimento da gestão do Sistema Único de Saúde, no âmbito da Avaliação de Tecnologias em Saúde (ATS) ${ }^{1,2,3}$.

O tempo médio de permanência de pacientes em ventilação mecânica (VM) invasiva na unidade de terapia intensiva é de 6,21 $( \pm 8,06)$ dias com mediana de 2 dias (referência). Maior tempo de permanência em VM está associado a um maior risco de infecções pneumônicas causadas por microrganismos resistentes, maior mortalidade e aumento dos custos. Por estas razões, recomenda-se a utilização de medidas para a redução do tempo de ventilação mecânica e a alta hospitalar o mais breve possível, tendo em vista que, mantendo o paciente internado, maiores serão as oportunidades de ele entrar em contato com microrganismos através de transmissão cruzada, ou de realizar mais procedimentos ou receber dispositivos invasivos, ocasionalmente, conferindo-lhe menor resistência à colonização e infecção ${ }^{3,4,5}$ 6,7,8.

A monitorização da mecânica ventilatória é recomendada como rotina para os pacientes em VM, seja ela não invasiva ou invasiva, e para isso se faz necessária a extração de uma série de dados que exigem a mensuração de vários parâmetros ventilatórios e gasométricos ${ }^{7}$. 
A Tomografia de Impedância Elétrica (TIE) surge como ferramenta alternativa aos métodos convencionais e invasivos de monitorização da função pulmonar e da mecânica ventilatória em tempo real e à beira do leito, na medida em que possibilita a equipe, a partir da análise de imagens em um monitor, a identificação e intervenção precoce em caso de complicações ventilatórias, como o pneumotórax e o derrame pleural que, juntamente com a Pneumonia Associada a Ventilação Mecânica (PAVM), são complicações comuns em paciente em $\mathrm{VM}^{5,7}$.

Ainda que sem consenso, estudos têm demonstrado a segurança e eficácia do uso do TIE à beira do leito para auxiliar na condução de manobras de recrutamento alveolar, frequentemente necessárias em casos de hipoxemia refratária em pacientes com Insuficiência Respiratória Aguda (IRA) e Síndrome do Desconforto Respiratório Agudo (SDRA), bem como a possibilidade de outras aplicações, além do acompanhamento de mudanças na ventilação regional, e da distribuição de ventilação e mecânica pulmonar regional, além do monitoramento de perfusão pulmonar e de ventilação / perfusão distribuição $8,9,10,11$.

A problemática do estudo é que, mesmo ainda não existindo evidências e consenso sobre a sua eficácia e efetividade e ainda que o uso da tecnologia não esteja um procedimento coberto pela tabela de reembolso do Sistema Único de Saúde (SUS) e nem conste no rol de procedimentos da Agência Nacional de Saúde (ANS), pressões para sua incorporação vêm se intensificando ${ }^{12,13}$.

O objetivo do estudo foi avaliar a efetividade do uso do TIE para a monitorização da função pulmonar e da mecânica ventilatória à beira do leito de pacientes em ventilação mecânica invasiva em unidades de terapia intensiva.

\section{Descrição da tecnologia}

No Brasil, ainda são poucos os estudos com o TIE, mas essa tecnologia já está sendo estudada há muitos anos. Profissionais de saúde e pesquisadores estão analisando diferentes abordagens e dispositivos alternativos para 0 monitoramento da função pulmonar e dinâmica ventilatória para orientar a tomada de decisões clínicas no suporte ventilatório ${ }^{11,12,14}$.

O TIE pode ser utilizado para visualizar continuamente a função pulmonar à beira do leito e para avaliar instantaneamente os efeitos das manobras terapêuticas sobre a dinâmica ventilatória e na distribuição ventilatória regional ${ }^{14,15,16,17,18}$.

O exame baseia-se nas diferenças existentes nas propriedades elétricas geradas por alterações do conteúdo de ar em pequenas regiões pulmonares, criando uma relação de impedância entre tais 
regiões. Para a realização de medições de bioimpedância, um conjunto reator de 16 eletrodos é colocado em torno da parede torácica do paciente, com um eletrodo adicional de referência, ligado a um ponto central no corpo, preferencialmente, no abdômen. $O$ eléctrodo de referência garante que todas as medições em diferentes pares de eletrodos são referenciadas ao mesmo potencial elétrico $^{16}$.

O equipamento determina então a distribuição da bioimpedância intratorácica por aplicação de uma corrente alternada conhecida "I1" a um primeiro par de eletrodos, medindo em seguida os potenciais de superfície resultantes " $\mathrm{Vn}$ " nos restantes dos 13 pares de eletrodos. São criados perfis de tensão usados para reconstruir uma imagem TIE em corte transversal que, com a ajuda de algoritmos específicos, vão gerar uma imagem dos pulmões, na tela do monitor. Para a interpretação clínica, o profissional deverá considerar que, na imagem do monitor, os Pixels gerados representam alterações do percentual de bioimpedância local comparado com uma referência obtida no início da aquisição da imagem ${ }^{16}$.

\section{Tecnologias Concorrentes Complementares}

O TIE não substitui inteiramente outros métodos de diagnóstico por imagem, como a Tomografia Computadorizada (TC), a Ecografia e os exames de raios- $\mathrm{X}$, métodos mais utilizados para avaliação da caixa torácica e do pulmão. A TC e a Ecografia Torácica (Ultrassom de Tórax) são, na atualidade, os dois métodos de imagens recomendados pelas Diretrizes Brasileiras de Ventilação Mecânica, para avaliar as condições do tórax e dos pulmões de pacientes em ventilação mecânica invasiva, como métodos de monitorização regional da mecânica ventilatório à beira do leito ${ }^{3}$.

Segundo as Diretrizes Brasileiras de Ventilação Mecânica, a TC deve ser utilizada como método diagnóstico nos casos de Insuficiência Respiratória de origem não clara, e usar a AngioTomografia na suspeita de Embolia Pulmonar. Em relação a ultrassonografia torácica, a referida Diretriz recomenda que, nos centros onde a tecnologia se encontra disponível, treinamento da equipe deverá ser feito para usar o ultrassom do tórax na identificação precoce de pneumotórax, derrame pleural e como auxiliar no procedimento terapêutico ${ }^{3}$.

\section{Situação da Tecnologia TIE no País}

Apesar da monitorização por tomografia de impedância elétrica pulmonar não ser um procedimento coberto pela tabela de reembolso do SUS e não constar no rol de procedimentos da ANS, é um procedimento recomendado pela Diretriz Brasileira de Ventilação Mecânica, desde 
2013, como métodos de monitorização regional para a detecção de alterações da ventilação pulmonar, como o pneumotórax, posição de tubo endotraqueal, alterações da ventilação conforme 0 decúbito, avaliação de recrutamento, colapso pulmonares e distribuição regional da ventilação, sugerindo que, futuramente, poderá vir a ser recomendado para a monitorização da perfusão pulmonar à beira do leito, de forma contínua.

No Brasil, a Drager Indústria e Comércio LTDA conseguiu junto a Agência de Vigilância Sanitária (ANVISA), em 2012, o registro do PulmoVista $500 \AA$ (Processo 25351.047079/2012-73), vencido em 26 de março de 2017. Este foi o único registro de tomógrafo de impedância elétrica encontrado na ANVISA (Registro 10407370096), como sistema de monitoração de paciente, que pode ser utilizado à beira do leito para a realização de tomografia de impedância elétrica pulmonar.

Existe ainda um equipamento nacional, O ENLIGHT® 1800 que é um equipamento criado, desenvolvido $e$ comercializado pela TIMPEL S.A., e fabricado pela Sérdia Eletrônica Industrial Ltda. O equipamento é destinado a ser operado por médicos, fisioterapeutas respiratórios e enfermeiros, que tenham recebido treinamento adequado pela TIMPEL ou representante habilitado, que tenham lido e entendido o manual de usuário e outras instruções de uso, e que sejam familiarizados com fisiologia pulmonar.

\section{Método}

Trata-se de um Parecer Técnico Científico construído a partir de uma revisão sistemática da literatura. A questão de pesquisa, baseada no acrônimo PICO, ficou assim estruturada para orientar a estratégia de busca na revisão:

População: Pacientes adultos internados em unidades de terapia intensiva submetidos à ventilação mecânica invasiva. Intervenção: uso do tomógrafo de impedância elétrica para a monitorização da função pulmonar, da mecânica ventilatória e do recrutamento alveolar. Comparação: técnicas convencionais de monitorização da função pulmonar e da mecânica ventilatória Desfechos: otimização da ventilação mecânica (otimização da Pressão Positiva ao Final da Expiração - PEEP e do recrutamento alveolar), complicações associadas à ventilação mecânica, tempo de permanência em ventilação mecânica (desabituação do ventilador).

\section{Estratégia de busca e seleção dos estudos}

Durante o mês de agosto de 2017, realizou-se uma busca na literatura científica, por estudos nas bases da Cochrane Library, MEDLINE via Portal 
PUBMED, BVS, ECRI, PEDRo, EMBASE, Banco de Teses e Dissertações da CAPES e Google Scholar.

Os Descritores, MeSH e ENTREE e seus respectivos sinônimos, utilizados na construção da estratégia de busca e recuperação das informações a partir do título e abstract dos artigos, encontram-se descritos no quadro 1 (nos anexos).

Foram critérios de inclusão, estudos envolvendo seres humanos de ambos os sexos, com idade igual ou superior a 18 anos, disponíveis em textos completos, nos idiomas inglês, espanhol e português, filtrados para revisão, revisão integrativa, revisões sistemáticas, ensaio clínico pragmático, estudo observacional, estudos de avaliação, relatos de casos, estudo comparativo, estudo multicêntrico, publicado nos últimos 10 anos.

As estratégias de busca foram estruturadas de forma a ser especialmente sensivel usando a estrutura de termos e sinônimo utilizada no MEDLINE, na BVS e no EMBASE (quadro 2).

As etapas da revisão foram: Identificação, Seleção, Elegibilidade e Inclusão. Um total de 89 estudos foram avaliados por pares de revisores, após eliminados os duplicados. Desse total, apenas 14 estudos foram incluídos no PTC (figura 1, nos anexos).

\section{Resultados}

Foram extraídas dos estudos, as seguintes informações: autores e ano de publicação, tipo de estudo, população, intervenção, desfechos e resultados e descritos no quadro 4 (nos anexos).

A qualidade dos estudos, foi avaliada de acordo com as recomendações das Diretrizes Metodológicas para elaboração de Pareceres Técnico-Científicos do Ministério da Saúde, que recomenda a utilização da escala de avaliação do nível de evidência da Oxford Centre for Evidence Based Medicine.

Os estudos de caso e as revisões foram avaliados como Grau de Recomendação C e Nível de Evidência 4. Os estudos observacionais foram avaliados como Grau de Recomendação B e Nível de Evidência 3B. A revisão sistemática foi avaliada como Grau de Recomendação B Nível de Evidência $3 A$ (quadro 3, nos anexos).

Dos 14 estudos incluídos, nenhum deles avaliou diretamente (head-to-head) o desempenho desta tecnologia em comparação com outras tecnologias disponíveis, para os desfechos avaliados neste PTC. Todos são descritivos, sendo 06 revisões e 05 estudos de caso, 02 estudos observacionais prospectivos e uma revisão sistemática, sem meta-análise, apresentavam diferenças na população, na comparação, nos desfechos e não 
respondiam diretamente à pergunta de interesse da revisão e foram publicados entre 2011 e 2017. Apenas dois estudos são nacionais.

Os estudos incluídos tinham como objetivo principal, avaliar o desempenho do tomógrafo de impedância elétrica na monitorização da resposta a intervenções terapêuticas durante a ventilação mecânica invasiva, incluindo mudança no modo de ventilação, reposicionamento do paciente, aspiração traqueal, manobras de recrutamento alveolar e alteração da pressão positiva expiratória final (PEEP) em pacientes, em comparação com diversos comparadores. O quadro 3 (nos anexos) apresenta uma síntese dos estudos selecionados.

A qualidade geral da evidência foi avaliada pelo Grading of Recommendations Assessment, Development and Evaluation (GRADE) e foi considerada como muito baixa (tabela 1 , nos anexos).

$\mathrm{Na}$ revisão de literatura realizada por Luecke et $a^{R 2}$, os autores concluíram que a tomografia computadorizada quantitativa é o padrão-ouro para avaliar a morfologia pulmonar. Entretanto, para monitorar o recrutamento e a hiperinflação do tecido pulmonar em diferentes níveis de pressão de inflação em doentes submetidos à ventilação mecânica invasiva, o tomógrafo de impedância elétrica e a ultrassonografia estão emergindo como ferramentas não invasivas valiosas, sem radiação, e que devem ser usadas em conjunto com parâmetros globais como mecânica pulmonar e troca de gás para adquirir informações adicionais sobre recrutamento e distribuição de ventilação, sobretudo quando se utiliza pressão positiva ao final da expiração (PEEP).

Karsten et $a l^{15}$ concluíram que é improvável que o TIE possa competir com tomografia computadorizada em imagens anatômicas, uma vez que espacialmente, a resolução não pode ser alcançada. Entretanto, o TIE parece apresentar melhores resultados no que se refere à alta resolução temporal, diante das mudanças tão dinâmicas no pulmão e a consequente variação da distribuição da ventilação, que pode ser medida facilmente à beira do leito com o TIE, sendo portanto uma tecnologia adequada para monitorar respiração e a ventilação regional, fornecendo aos profissionais, informações imediatas sobre o impacto da alteração das configurações do ventilador, o que não é possível utilizando imagens de tomografia ou $\mathrm{RX}$ de tórax.

O uso do TIE na prática clínica ainda é incomum e outros dados sobre a interpretação dos seus resultados e a devida configuração clínica, ainda são necessárias ${ }^{30,16,22}$.

Na tentativa de testar a hipótese de que nem todos os pacientes com tecido pulmonar recrutado de forma apreciável durante uma manobra de recrutamento 
mostram melhora significativa da oxigenação, Yun et $a \beta^{\beta 3}$, através de um estudo observacional prospectivo, combinaram a tomografia de impedância elétrica com medidas de oxigenação para examinar as discrepâncias da ventilação pulmonar e perfusão versus oxigenação após a realização de manobras de recrutamento. No estudo, conduzido 20 pacientes com síndrome de dificuldade respiratória aguda de janeiro de 2014 a dezembro de 2014, um teste de PEEP decrescente foi realizado para selecionar o nível PEEP após as manobras.

O potencial do tomógrafo de impedância elétrica também foi destacado na revisão realizada por Moerer et $a^{R 3}$. Concluíram que a aplicação clínica do TIE requer padronização quanto às manobras respiratórias terapêuticas que são aplicadas. Abordagens quantitativas semelhantes para interpretar dados do TIE precisam de um quadro harmonizado, que atualmente, ainda não é o caso, o que limita o progresso na aplicação clínica desta tecnologia.

Em uma recente revisão realizada por Theerawit et $a^{\beta 2}$ sobre as mais recentes alternativas para monitorização respiratória de pacientes adultos em unidades de terapia intensiva, submetidos a ventilação mecânica invasiva, concluíram que a técnica do TIE ainda é susceptível a interferências por várias fontes incluindo a ventilação de alta frequência e monitorização hemodinâmica invasiva. Ainda, que melhorias tecnológicas precisam ser realizadas para garantir a eficácia e efetividade desta técnica na prática clínica diária.

Walsh $^{33}$ descreveram em uma revisão, que o TIE tem sido útil também na detecção de pneumotórax, na quantificação de edema pulmonar e na comparação da distribuição de ventilação entre diferentes modos de ventilação mecânica. Concluíram, entretanto, chamando a atenção para a necessidade de pesquisas de comparações diretas com outros métodos existentes para provar a viabilidade do uso contínuo do equipamento em pacientes em ventilação mecânica, como uma ferramenta de cabeceira útil na UTI.

Kobylianskii et a ${ }^{\beta 4}$ concluíram que em pacientes adultos, 0 tomógrafo de impedância elétrica foi validado com sucesso pelos estudos, para avaliação da distribuição de ventilação, medição de alterações no volume pulmonar, estudo da mecânica respiratória regional e pesquisa de parâmetros não ventilatórios. A tomografia de impedância elétrica também demonstrou ser útil no monitoramento de alterações regionais do sistema respiratório durante intervenções em ventilação mecânica invasiva, embora a literatura existente não tenha evidência de resultado clínico. 


\section{Conclusão}

A utilização do tomógrafo de impedância elétrica nas unidades de terapia intensiva parece capaz de fornecer informações semelhantes aquelas fornecidas pela tomografia computadorizada do tórax, padrão-ouro atual, com a vantagem de disponibilizar imagens em tempo real à beira do leito do paciente, sem a necessidade de exposição ou a necessidade de transferir o paciente e sem radiação, podendo beneficiar pacientes em diversas condições clínicas que necessitem de ventilação mecânica invasiva.

O uso do Tomógrafo por Impedância Elétrica Pulmonar à beira do leito pode no futuro se tornar uma importante ferramenta clínica alternativa, capaz de guiar, a cada movimento respiratório, possíveis ajustes da ventilação regional e orientando decisões por manobras de recrutamento alveolar, em pacientes com síndrome do desconforto respiratório agudo, auxiliando a equipe a refinar a escolha da pressão positiva expiratória final ideal pósrecrutamento máximo, considerando a quantificação de variáveis possivelmente deletérias, como o grau de colapso e hiperdistensão alveolar.

As evidências sumarizadas nesse PTC mostraram que ainda são necessárias a padronização da monitorização/avaliação atualmente aplicada com o uso do tomógrafo de impedância elétrica, de modo que a técnica possa atender aos requisitos necessários e essenciais para uma aplicação clínica eficaz e efetiva.

Com relação ao domínio econômico, não foi possível fazer qualquer consideração nesse PTC, tendo em vista que nenhum dos estudos incluídos abordavam tal domínio. Tentamos contato via e-mail e telefone com as empresas TIMPEL S.A. e SINALVITAL, que comercializam o tomógrafo de impedância elétrica ENLIGHT® 1800, fabricado pela Sérdia Eletrônica Industrial Ltda, na tentativa de conseguirmos informações sobre o custo do equipamento, que possibilitasse a abordagem do domínio econômico nesse PTC, mas não obtivemos o retorno das empresas.

Este PTC deve ser considerado à luz de suas limitações. Primeiro, artigos pertinentes podem ter sido omitidos nos resultados de nosso banco de dados devido à seleção dos termos de pesquisa e as bases pesquisadas. Em segundo lugar, apenas estudos publicados na língua portuguesa, inglesa e espanhola foram incluídos. Em terceiro lugar, os detalhes relacionados às questões técnicas estão além do escopo deste PTC e por isso, não foram abordados. Finalmente, os estudos incluídos compartilham limitações, comum como a baixa qualidade metodológica, a falta de um comparador e o pequeno tamanho amostral, comprometendo e 
limitando consideravelmente a validade interna e externa.

Portanto, diante da escassez de estudos que avaliaram a efetividade do tomógrafo de impedância elétrica na monitorização de função pulmonar e dinâmica respiratório de pacientes em ventilação mecânica invasiva em comparação com as técnicas convencionais, bem como a pouca robustez daqueles que foram incluídos nesse PTC, não recomendamos esta tecnologia, sendo necessários estudos com evidência mais robusta e metodologias bem definidas a fim de avaliar se esta tecnologia poderia otimizar a ventilação mecânica, reduzir o tempo para desabituação do ventilador e reduzir as complicações associadas à ventilação mecânica.

\section{Financiamento}

Este estudo foi financiado com recursos do Ministério da Saúde, via PROADISUS e Hospital Alemão Oswaldo Cruz - SP.

\section{Referências}

1- Brasil. Ministério da Saúde. Portaria no2510/GM, 19 dez 2005. [Internet]. Disponível em: http://bvsms.saude.gov.br/bvs/saudelegis/g m/2005/prt2510_19_12_2005.html 2- Brasil. Ministério da Saúde. Secretaria de Ciência, Tecnologia e Insumos Estratégicos. Departamento de Ciência e Tecnologia. Diretrizes metodológicas: elaboração de estudos para avaliação de equipamentos médicos assistenciais / Ministério da Saúde, Secretaria de Ciência, Tecnologia e Insumos Estratégicos, Departamento de Ciência e Tecnologia.

Ministério da Saúde [Internet]. 2013 Jun [citado 2017 Mai 17]; Disponível em: http://bvsms.saude.gov.br/bvs/publicacoes/ diretrizes_metodologicas_elaboracao_estud os.pdf

3- Brasil. Ministério da Saúde. Secretaria de Ciência, Tecnologia e Insumos

Estratégicos. Departamento de Ciência e Tecnologia. Diretrizes metodológicas: elaboração de pareceres técnico-científicos revisada e atualizada [Internet] 2013. [citado 2017 Mai 17]; 4. ed. Disponível em: http://bvsms.saude.gov.br/bvs/publicacoes/ diretrizes_metodologicas_elaboracao_estud os.pdf

4- Blankman P, Gommers D. Lung monitoring at the bedside in mechanically ventilated patients. Curr Opin Crit Care [Internet]. 2012 Jun [citado 2017 Mai 17];18(3):261-6. Disponível em: https://www.ncbi.nlm.nih.gov/pubmed/2254 3298

5- Anderson J. et col. Noninvasive Positive Pressure Ventilation in Patients with Acute Respiratory Failure after Tracheal Extubation, Revista Brasileira de Terapia Intensiva [Internet] 2006. Dez [citado 2017 Set 4];18(4):338-43. Disponível em: https://www.ncbi.nlm.nih.gov/pubmed/2531 0546

6- Arad M, Zlochiver S, Davidson T, Shoenfeld Y, Adunsky A, Abboud S. The detection of pleural effusion using a parametric EIT technique. Physiol Meas [Internet] 2009 Apr [citado 2017 Set 4];30(4):421-8. Disponível em: https://www.ncbi.nlm.nih.gov/pubmed/1933 2893doi: 10.1088/0967-3334/30/4/006 7- Barbas CSV, Ísola AM, Farias AMC, Cavalcanti AB, Gama AMC, Duarte ACM et al. Brazilian recommendations of mechanical ventilation 2013. Part I. Rev. bras. ter. intensiva [Internet]. 2014 Jun [citado 2017 Feb 17];26 (2): 89-121.

Disponível em:

http://www.scielo.br/scielo.php?script=sci_a rttext\&pid=S0103507X2014000200089\&Ing $=$ en

8- Brasil. Ministério da Saúde. Secretaria de Ciência, Tecnologia e Insumos Estratégicos. Departamento de Ciência e Tecnologia. Diretrizes metodológicas: 
Sistema GRADE - manual de graduação da qualidade da evidência e força de recomendação para tomada de decisão em saúde. / Ministério da Saúde, Secretaria de Ciência, Tecnologia e Insumos

Estratégicos, Departamento de Ciência e Tecnologia - Brasília: Ministério da Saúde, 2014.

9- Costa ELV, Chaves CN, Gomes S, et al. Real-time detection of pneumothorax using electrical impedance tomography. Crit Care Med 2008; 36:1230-1238.

10- Gattinoni L, Pesenti A, Bombino M, Baglioni S, Rivolta M, Rossi F, Rossi G, Fumagalli R, Marcolin M, Mascheroni D, Torresin A. Relationships between lung computed tomographic density, gas exchange, and PEEP in Acute Respiratory Failure. Anesthesiology 1988; 69: 824-832 11- Holder DS. Clinical and physiological applications of Electrical Impedance Tomography. UCL Press 1993; ISBN 1 85728-164-0 HB

12- Holder DS. Electrical Impedance Tomography Methods, History and Applications. IOP 2005; ISBN 0750309520 13- Howland, RH. Limitations of evidence in the practice of evidence-based medicine. Journal of Psychosocial Nursing, [S.I.], v. 45, n. 11, nov. 2007.

14- lotti GA, Braschi A. Monitorização da mecânica respiratória. São Paulo: Atheneu; 2004.

15- Karsten J, Bohlmann MK, SedemundAdib, et al. Electrical impedance tomography may optimize ventilation in a postpartum woman with respiratory failure[J]. International Journal of Obsteric Anesthesia, 2013, 22(1): 67-71.

16- Karsten J, Grusnick C, Paarmann H, HeringlakeM, Heinze $\mathrm{H}$. Positive endexpiratory pressuretitration at bedside using electrical impedancetomography in postoperative cardiac surgerypatients. Acta Anaesthesiologica Scandinavica. Disponível em: <

https://www.ncbi.nlm.nih.gov/pubmed/2586 7049 >. Acesso em: 2017 mai. 2017. 17- Kotani T, Tanabe H, Yusa H, Saito S, Yamazaki K, Ozaki M. Electrical impedance tomography-guided prone positioning in a patient with acute cor pulmonale associated with severe acute respiratory distress syndrome. J Anesth. 2016 Feb;30(1):161-5. Epub 2015 Oct 7. Disponível em: < https://www.ncbi.nlm.nih.gov/pubmed/2644 6805>. Acesso em: 2017 mai. 2017. 18- Leonhardt S, Lachmann B. Electrical impedance tomography: the holygrail of ventilation and perfusion monitoring? Intensive Care Med. 2012;38(12):1917-29. 19- Lisboa DDJ, Medeiros EF, Alegretti LG, Badalotto D, Maraschin. Profile of patients in invasive mechanical ventilation in intensive care unit J. Biotec. Biodivers. v. 3, N.1: pp. 18-24, Fev. 2012

20- Lopes FA, et al. Pendelluft diagnosed from ventilator weaning indexes obtained through bioelectrical impedance tomography: a case report. Sao Paulo Med. J., São Paulo, 2017. Disponível em: <http://www.scielo.br/scielo.php?script=sci arttext\&pid=S1516-

$31802017005004104 \& \operatorname{lng}=e n \& n r m=i s o>$. Acesso em: 2017 mai. 2017.

21- Loss SH, Oliveira RP, Maccari JG, Savi A, Boniatti MM, Hetzel MP et al. The reality of patients requiring prolonged mechanical ventilation: a multicenter study. Rev. bras. ter. intensiva [Internet]. 2015. Disponível em:

$<\mathrm{http}: / /$ www.scielo.br/scielo.php?script=sci arttext\&pid=S0103-

$507 X 2015000100026 \& \operatorname{lng}=e n>$. Acesso em: 2017 mai. 2017.

22- Luecke T, Corradi F, Pelosi P. Lung imaging for titration of mechanical ventilation. Curr Opin Anaesthesiol. 2012 Apr;25(2):131-40. Disponível em: < https://www.ncbi.nlm.nih.gov/pubmed/2239 5440> Acesso em: 2017 mai. 2017. 23- Moerer O; Hahn G; Quintel M. Lung impedance measurements to monitor alveolar ventilation. Current Opinion in Critical Care: June 2011 - Volume 17 Issue 3 - p 260-267. Disponível em: < https://www.ncbi.nlm.nih.gov/pubmed/2147 8747 >. Acesso em: 2017 mai. 2017. 24- Obyllanskii J, Murray Alistair, Brace Debbie, Go-ligher Ewan, Fan Eddy, Electrical Impedance Tomography in Adult Patients Under-going Mechanical Ventilation: A Systematic Review. J Crit Care. 2016 Oct; 35:33-50. Epub 2016 May 
3. Disponível em: <

https://www.ncbi.nlm.nih.gov/pubmed/2748

1734>. Acesso em: 2017 mai. 2017.

25- Pinsky MR. Heart lung interactions during mechanical ventilation. Current Opinion in Critical Care: June 2012 -

Volume 18 - Issue 3 - p 256-260.

Disponível em: <

https://www.ncbi.nlm.nih.gov/pubmed/2247

3256 >. Acesso em: 2017 mai. 2017.

26- Pongdhep T, Yuda S, Lorenzo B; Paolo

$P$. Respiratory monitoring in Adult Intensive

Care Unit, Expert Review of Respiratory

Medicine. Disponível em: <

http://ieeexplore.ieee.org/abstract/document /7877921/ >. Acesso em: 2017 mai. 2017.

27- Rieira J. et al. Electrical impedance

tomography in acute lung injury. Med.

Intensiva, v. 35, n.8, p.509-517, nov.2011.

Disponível em:

<https://www.ncbi.nlm.nih.gov/pubmed/216 80060 >. Acesso em: 2017 mai. 2017.

28- Romero A, Alonso B, Latorre I, García

J. Respiratory monitoring with electrical impedance tomography for lung protective ventilation and alveolar recruitment maneuver in a patient with a single lung transplant and early graft dysfunction. Rev Esp Anestesiol Reanim. 2016 JunJul;63(6):347-52. doi:

10.1016/j.redar.2015.09.003. Epub 2015

Nov 27. Disponível em: <

https://www.ncbi.nlm.nih.gov/pubmed/2663

3603>. Acesso em: 2017 mai. 2017.

29- Rosa RG, Rutzen W, Madeira L, Ascoli

AM, Dexheimer FL, Maccari JG, et al. Uso da tomografia por impedância elétrica torácica como ferramenta de auxílio às manobras de recrutamento alveolar na síndrome do desconforto respiratório agudo: relato de caso e breve revisão da literatura. Rev Bras. Ter. Intensiva. 2015;27(4):406-411. Disponível em: <http://www.scielo.br/pdf/rbti/v27n4/0103507X-rbti-27-04-0406.pdf>. Acesso em: 2017 mai. 2017.

30- Rudnicki SM, Gaszyński T, Gaszyński

W. Assessment of regional ventilation in acute respiratory distress syndrome by electrical impedance tomography.

Anaesthesiol Intensive Ther. 2015;47(1):7781. Disponível em: < https://www.ncbi.nlm.nih.gov/pubmed/2575 1294>. Acesso em: 2017 mai. 2017. 31- Sociedade Paulista de Infectologia. Diretrizes sobre Pneumonia Associada a Ventilação Mecânica (PAV). São Paulo Office; 2006.

32-Theerawit $P$, Sutherasan $Y$, Ball $L$, Pelosi P.Respiratory monitoring in adult intensive care unit. Expert Rev Respir Med. 2017 Jun;11(6):453-468. doi: 10.1080/17476348.2017.1325324. Epub 2017 May 10. Disponível em:< https://www.ncbi.nlm.nih.gov/pubmed/2845 2241 >. Acesso em: 2017 mai. 2017. 33- Walsh BK; Smallwood CD. Electrical Impedance Tomography During Mechanical Ventilation. Respiratory Care Oct 2016, 61 (10) 1417-1424. Disponível em:< http://rc.rcjournal.com/content/61/10/1417>. Acesso em: 2017 mai. 2017.

34- Kobylianskii J, Murray A, Brace D, Goligher E, Fan E. (2016). Electrical impedance tomography in adult patients undergoing mechanical ventilation: $A$ systematic review. Journal of Critical Care, 35, 33-50. doi:10.1016/j.jcrc.2016.04.028 Disponível em: <

https://daneshyari.com/article/preview/2764 434.pdf>. Acesso em: 2017 mai. 2017. 35- Yun L; He H; Moller K; Frerichs I; Liu Dawei; Zhao Z. Assessment of Lung Recruitment by Electrical Impedance Tomography and Oxygenation in ARDS Patients. Disponível em: < https://www.ncbi.nlm.nih.gov/pmc/articles/P MC4900735/ > . Acesso em: 2017 mai. 2017.

36- Victorino JA, Borges JB, Okamoto VN, Matos GF, Tucci MR, Caramez MP, et al. Imbalances in regional lung ventilation: a validation study on electrical impedance tomography. Am J Respir Crit Care Med. 2004;169(7):791-800.

37- Larkin J, Ascierto PA, Dréno B, Atkinson V, Liszkay G, Maio M, et al. Combined vemurafenib and cobimetinib in BRAF-mutated melanoma. N Engl J Med [Internet]. 2014 Nov [citado 2019 Apr 4];371(20):1867-76. Disponível em: https://www. nejm.org/doi/full/10.1056/NEJMoa1408868. doi: 10.1056/NEJMoa1408868 


\section{Anexos}

Quadro 1: Descritores, Mesh e ENTREE utilizados na estratégia de busca para cada braço do PICO

\begin{tabular}{|c|c|c|c|c|c|}
\hline PICO & DESCRITORES & SINÔNIMOS & MeSH & ENTRY TERMS & EMTREE \\
\hline 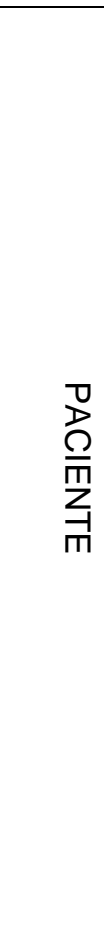 & $\begin{array}{l}\text { Pacientes } \\
\text { Internados; } \\
\text { Respiração } \\
\text { Artificial; } \\
\text { Cuidados } \\
\text { Críticos; } \\
\text { Insuficiência } \\
\text { respiratória }\end{array}$ & $\begin{array}{l}\text { Ventilação } \\
\text { Mecânica; } \\
\text { Cuidado } \\
\text { Cirúrgico } \\
\text { Intensivo; } \\
\text { Cuidado } \\
\text { Intensivo } \\
\text { Cirúrgico; } \\
\text { Cuidado } \\
\text { Intensivo; } \\
\text { Cuidados } \\
\text { Cirúrgicos } \\
\text { Intensivos; } \\
\text { Cuidados } \\
\text { Intensivos; } \\
\text { Cuidados } \\
\text { Intensivos } \\
\text { Cirúrgicos; } \\
\text { Terapia } \\
\text { Intensiva; } \\
\text { Terapia } \\
\text { Intensiva } \\
\text { Cirúrgica }\end{array}$ & $\begin{array}{l}\text { Inpatients; } \\
\text { Respiration, } \\
\text { Artificial, } \\
\text { Critical Care, } \\
\text { Respiratory } \\
\text { Insufficiency }\end{array}$ & 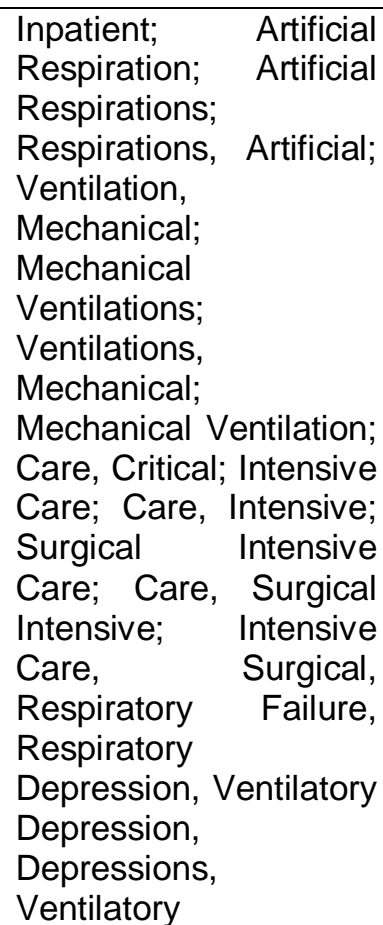 & $\begin{array}{l}\text { Inpatients; } \\
\text { Respiration, } \\
\text { Artificial, } \\
\text { Critical Care, } \\
\text { Respiratory } \\
\text { Insufficiency }\end{array}$ \\
\hline 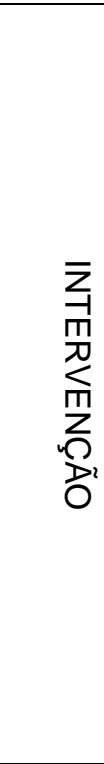 & $\begin{array}{l}\text { Impedância } \\
\text { Elétrica; } \\
\text { Tomografia }\end{array}$ & $\begin{array}{l}\text { Impedância } \\
\text { Bioelétrica; } \\
\text { Resistência } \\
\text { Elétrica; } \\
\text { Impedância; } \\
\text { Resistência } \\
\text { Ôhmica }\end{array}$ & $\begin{array}{l}\text { Electrical } \\
\text { impedance } \\
\text { tomography; } \\
\text { Electric } \\
\text { Impedance; } \\
\text { Tomography; }\end{array}$ & 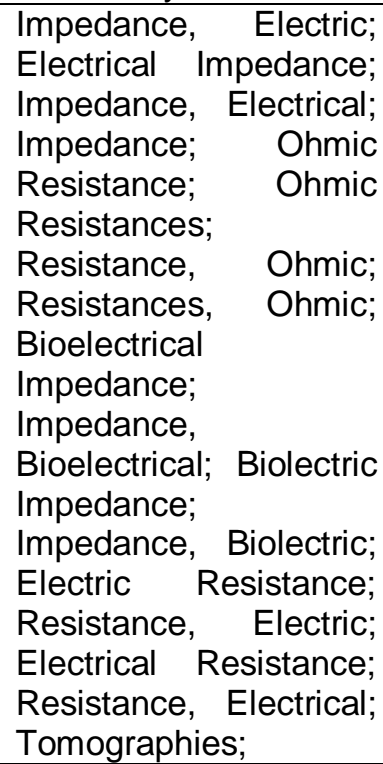 & $\begin{array}{l}\text { Electrical } \\
\text { impedance } \\
\text { tomography; } \\
\text { Electric } \\
\text { Impedance; } \\
\text { Tomography; }\end{array}$ \\
\hline
\end{tabular}

Fonte: os autores

Rev. Saúde Digital Tec. Educ., Fortaleza, CE, v. 5, n.1, p.16-36, jan./abr. 2020. 
Quadro 2: Estratégia de busca para a BVS, Banco de Teses e Dissertações da CAPES, Cochrane Library e PubMed/Medline

\begin{tabular}{|c|c|c|c|c|}
\hline PICO & BVS & $\begin{array}{c}\text { BANCO de TESES } \\
\text { da CAPES }\end{array}$ & COCHRANE & $\begin{array}{l}\text { MEDLINE/EMBAS } \\
\text { E }\end{array}$ \\
\hline 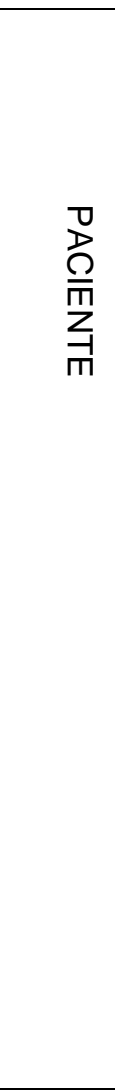 & $\begin{array}{lr}\# 1 & \text { Pacientes } \\
\text { Internados } & \text { AND } \\
\text { Cuidado } & \text { Cirúrgico } \\
\text { Intensivo } & \text { OR } \\
\text { Cuidado } & \text { Intensivo } \\
\text { Cirúrgico } & \text { OR } \\
\text { Cuidado } & \text { Intensivo } \\
\text { OR } & \text { Cuidados } \\
\text { Cirúrgicos } & \\
\text { Intensivos } & \text { OR } \\
\text { Cuidados } & \text { Intensivos } \\
\text { OR } & \text { Cuidados } \\
\text { Intensivos } & \\
\text { Cirúrgicos } & \text { OR } \\
\text { Terapia } & \text { Intensiva } \\
\text { OR } & \text { Terapia } \\
\text { Intensiva } & \text { Cirúrgica } \\
\text { OR } & \text { Respiração } \\
\text { Artificial } & \text { OR } \\
\text { Ventilação } & \text { Mecânica } \\
\text { OR } & \text { Cuidados } \\
\text { Críticos } & \end{array}$ & $\begin{array}{lr}\text { \#1 } & \text { Pacientes } \\
\text { Internados } & \text { AND } \\
\text { Cuidado } & \text { Cirúrgico } \\
\text { Intensivo } & \text { OR } \\
\text { Cuidado } & \text { Intensivo } \\
\text { Cirúrgico } & \text { OR } \\
\text { Cuidado } & \text { Intensivo } \\
\text { OR } & \text { Cuidados } \\
\text { Cirúrgicos } & \\
\text { Intensivos } & \text { OR } \\
\text { Cuidados } & \\
\text { Intensivos } & \text { OR } \\
\text { Cuidados } & \\
\text { Intensivos } & \\
\text { Cirúrgicos } & \text { OR } \\
\text { Terapia } & \text { Intensiva } \\
\text { OR } & \text { Terapia } \\
\text { Intensiva } & \text { Cirúrgica } \\
\text { OR } & \text { Respiração } \\
\text { Artificial } & \text { OR } \\
\text { Ventilação } & \\
\text { Mecânica } & \\
\text { Cuidados } & \text { Críticos }\end{array}$ & 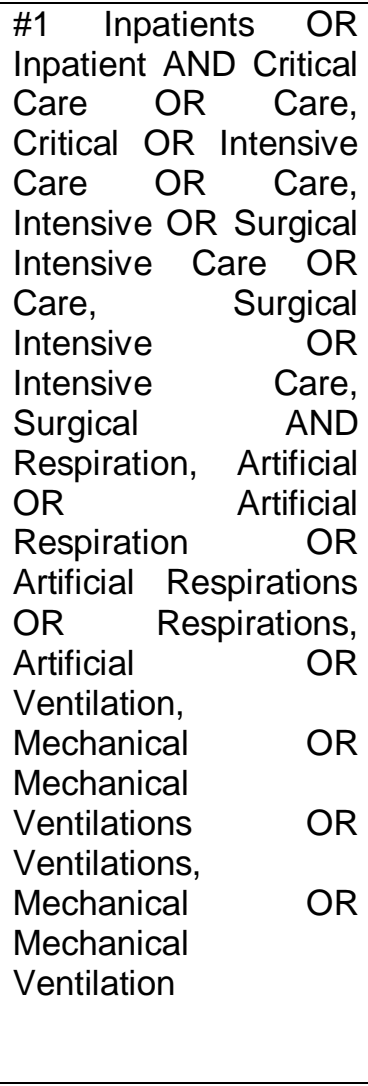 & 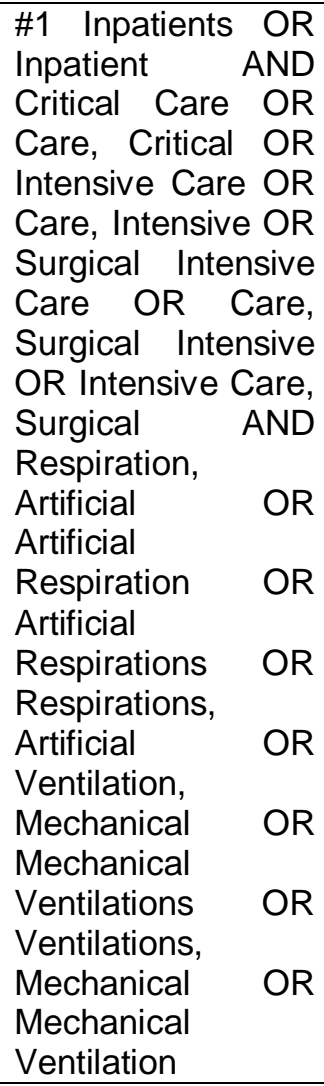 \\
\hline 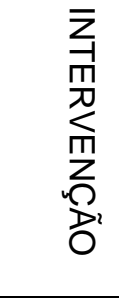 & $\begin{array}{l}\text { \#2 Tomografia de } \\
\text { impedância elétrica } \\
\text { pulmonar }\end{array}$ & $\begin{array}{l}\text { \#2 Tomografia de } \\
\text { Impedância } \\
\text { Elétrica pulmonar }\end{array}$ & $\begin{array}{l}\text { \#2 Pulmonary } \\
\text { Electric Impedance } \\
\text { Tomography OR } \\
\text { Electrical impedance } \\
\text { tomography }\end{array}$ & $\begin{array}{l}\text { \#2 Pulmonary } \\
\text { Electric Impedance } \\
\text { Tomography OR } \\
\text { Electrical } \\
\text { impedance } \\
\text { tomography }\end{array}$ \\
\hline $\begin{array}{l}\text { M } \\
\text { 羿 } \\
\frac{D}{1} \\
\frac{\Pi}{D}\end{array}$ & \#1 AND \#2 & \#1 AND \#2 & \#1 AND \#2 & \#1 AND \#2 \\
\hline 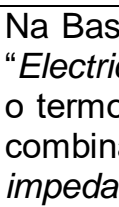 & L & (1) & pedância elétrica & $\begin{array}{l}\text { Ite, foi utilizado } \\
\text { Scholar, foram } \\
\text { ar"; "Pulmonary }\end{array}$ \\
\hline
\end{tabular}

Fonte: os autores

Rev. Saúde Digital Tec. Educ., Fortaleza, CE, v. 5, n.1, p.16-36, jan./abr. 2020. 
Figura 1: Fluxograma da seleção dos artigos - PRISMA Flow Diagram

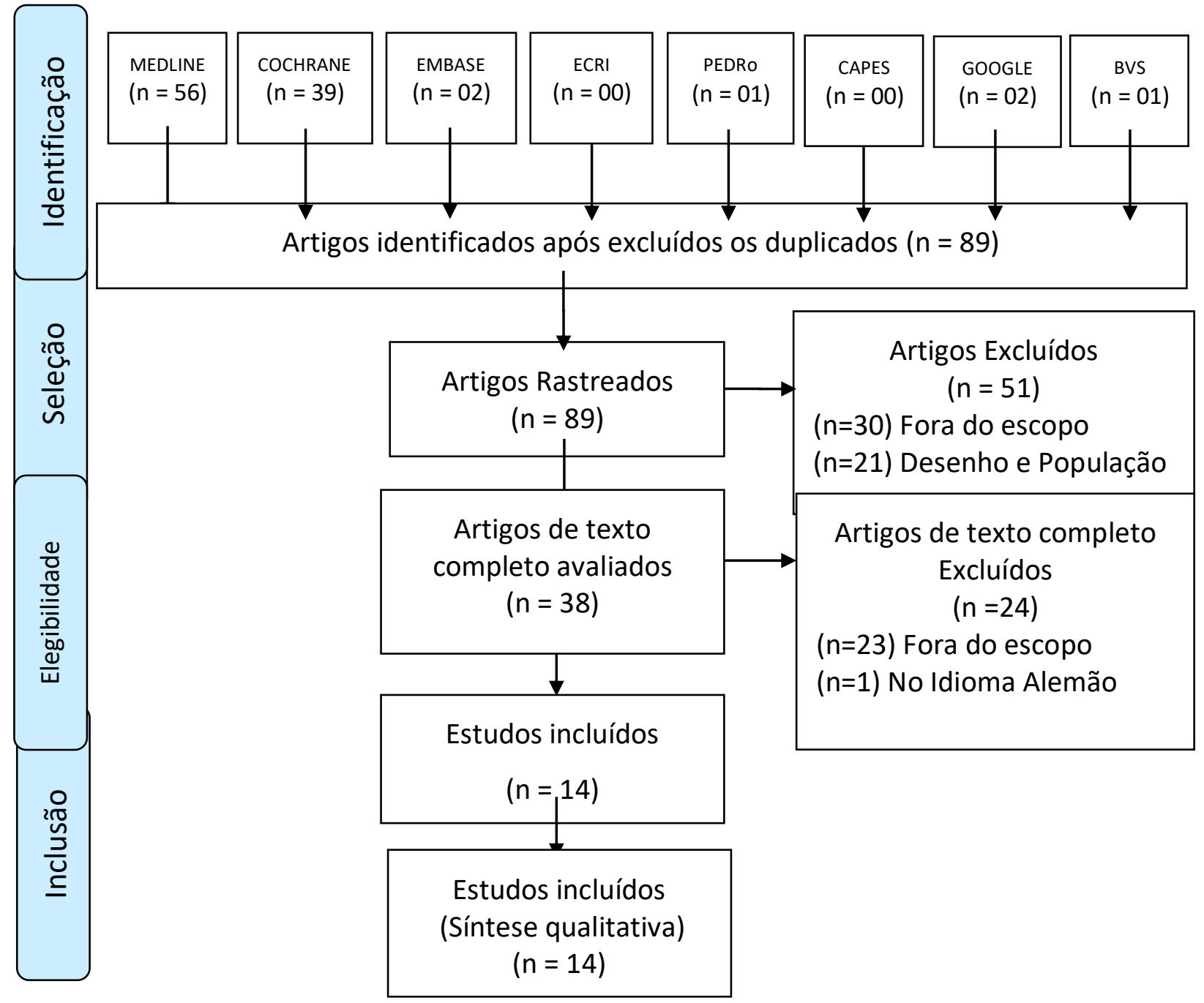

Fonte: os autores

Quadro 3: Classificação do nível de evidência dos estudos incluídos (Escala de Oxford)

\begin{tabular}{|c|c|c|c|}
\hline AUTOR & DESENHO & RECOMENDAÇÃO & EVIDÉNCIA \\
\hline J. Karsten et al, 2012 & Estudo de caso & GRAU C & NÍVEL 4 \\
\hline P. Blankman e D. Gommers, 2012 & Revisão & GRAU C & NÍVEL4 \\
\hline O. Moerer, G. Hahn e M. Quintel, 2011 & Revisão & GRAU C & NÍVEL 4 \\
\hline P. Theerawit et al, 2017 & Revisão & GRAU C & NIVEL4 \\
\hline L. Yun et al, 2016 & Observacional & GRAU B & NÍVEL 3B \\
\hline R. Rosa et al, 2015 & Estudo de caso & GRAU C & NÍVEL 4 \\
\hline T. Kotani et al, 2015. & Estudo de caso & GRAU C & NÍVEL 4 \\
\hline $\begin{array}{l}\text { M. Stankiewicz-Rudnicki, T. Gaszyński, W. } \\
\text { Gaszyński, } 2015\end{array}$ & Revisão & GRAU C & NÍVEL 4 \\
\hline B. Walsh e C. Smallwood, 2016 & Revisão & GRAU C & NÍVEL 4 \\
\hline J. Kobylianskii et al, 2016 & $\begin{array}{l}\text { Revisão } \\
\text { sistemática }\end{array}$ & GRAU B & NIVEL 3A \\
\hline $\begin{array}{l}\text { A. Romero, B. Alonso, I. Latorre, J. García, } \\
2015\end{array}$ & Estudo de caso & GRAU C & NÍVEL 4 \\
\hline J. Karsten et al, 2015. & Observacional & GRAU B & NÍVEL 3B \\
\hline T. Luecke, F. Corradi, P. Pelosib, 2012 & Revisão & GRAU C & NÍVEL 4 \\
\hline
\end{tabular}

Rev. Saúde Digital Tec. Educ., Fortaleza, CE, v. 5, n.1, p.16-36, jan./abr. 2020. 


\begin{tabular}{|l|l|l|l|}
\hline F. A Lopes et al, 2016 & Estudo de Caso & GRAU C & NÍVEL 4 \\
\hline
\end{tabular}

Fonte: os autores

Tabela 1: Avaliação da qualidade geral da evidência (GRADE)

\begin{abstract}
Tomógrafo de Impedância Elétrica Pulmonar comparado a técnicas convencionais para monitorização da função pulmonar e dinâmica respiratória
\end{abstract}

\begin{tabular}{|c|c|c|}
\hline Desfechos & $\begin{array}{l}\text { № de } \\
\text { participantes } \\
\text { (Estudos) } \\
\text { Seguimento }\end{array}$ & $\begin{array}{l}\text { Certeza da evidência } \\
\text { (GRADE) }\end{array}$ \\
\hline $\begin{array}{l}\text { Otimização da ventilação mecânica, PEEP } \\
\text { e recrutamento alveolar (PEEP) } \\
\text { avaliado com: não informado } \\
\text { seguimento: média } 5 \text { semanas }\end{array}$ & $\begin{array}{c}28 \\
\text { (11 estudos } \\
\text { observacionais) }\end{array}$ & $\begin{array}{c}\oplus \bigcirc \bigcirc \bigcirc \\
\text { MUITO BAIXA a,b }\end{array}$ \\
\hline $\begin{array}{l}\text { Complicações associadas à ventilação } \\
\text { mecânica (Complicações) } \\
\text { avaliado com: Não informado } \\
\text { seguimento: média } 5 \text { semanas }\end{array}$ & $\begin{array}{c}\text { (1 estudo } \\
\text { observacional) }\end{array}$ & $\begin{array}{l}\oplus \bigcirc \bigcirc \bigcirc \\
\text { MUITO BAIXA }\end{array}$ \\
\hline $\begin{array}{l}\text { Tempo de permanência em ventilação } \\
\text { mecânica (Tempo em Ventilação) } \\
\text { avaliado com: Não informado } \\
\text { seguimento: média } 5 \text { semanas }\end{array}$ & $\begin{array}{c}1 \\
\text { ( estudos } \\
\text { observacionais) }\end{array}$ & $\begin{array}{l}\oplus \bigcirc \bigcirc \bigcirc \\
\text { MUITO BAIXA a,b }\end{array}$ \\
\hline
\end{tabular}

\title{
Explicações
}

a. Um único caso foi descrito. Braço único.

b. Não teve comparador

c. Revisão narrativa

Fonte: os autores

Quadro 4: Síntese dos estudos incluídos no PTC.

\begin{tabular}{|c|c|c|c|c|}
\hline ESTUDOS & DESENHO/POPULAÇĀO & INTERVENÇĀO & DESFECHO & RESULTADOS \\
\hline $\begin{array}{l}\text { J. Karsten et } \\
\text { al, } 2012\end{array}$ & $\begin{array}{l}\text { Estudo de Caso. } \\
\text { Observação direta de } 01 \\
\text { mulher de } 32 \text { anos em } \\
\text { pós-parto com } \\
\text { insuficiência respiratória } \\
\text { por embolia amniótica }\end{array}$ & $\begin{array}{l}\text { Uso } \\
\text { PulmoVista; do } \\
\text { Drager Medical } \\
\text { para monitorar } \\
\text { PEEP }\end{array}$ & $\begin{array}{l}\text { Otimização } \\
\text { da PEEP }\end{array}$ & $\begin{array}{l}\text { Com o TIE, a PEEP foi } \\
\text { otimizada, permitindo } \\
\text { uma diminuição no FiO2. } \\
\text { Nas primeiras } 36 \text { h, } \\
\text { PEEP foi reduzido de } 15 \\
\text { a } 10 \text { mbar, permitindo o } \\
\text { início do desmame } \\
\text { respiratório. O paciente } \\
\text { foi extubado no final do } \\
\text { dia quatro }\end{array}$ \\
\hline \multicolumn{5}{|c|}{ Limitações: Único braço, sem comparador para o TIE } \\
\hline $\begin{array}{l}\text { P. Blankman e } \\
\text { D. Gommers, } \\
2012\end{array}$ & $\begin{array}{lr}\text { Revisão de } & \text { literatura. } \\
\text { Pacientes } & \text { com } \\
\text { insuficiência } & \text { respiratória }\end{array}$ & $\begin{array}{l}\text { Uso TIE para } \\
\text { monitorização } \\
\text { pulmonar }\end{array}$ & $\begin{array}{l}\text { Otimização } \\
\text { da ventilação }\end{array}$ & $\begin{array}{l}\text { O TIE provou ser seguro } \\
\text { e pode ser usado para } \\
\text { monitorar as alterações }\end{array}$ \\
\hline
\end{tabular}

Rev. Saúde Digital Tec. Educ., Fortaleza, CE, v. 5, n.1, p.16-36, jan./abr. 2020.

ISSN: 2525-9563 


\begin{tabular}{|c|c|c|c|c|}
\hline & $\begin{array}{ll}\text { aguda submetidos a } & \text { submeticana } \\
\text { ventilação } & \text { mecânica } \\
\text { invasiva } & \end{array}$ & & & $\begin{array}{l}\text { do volume pulmonar em } \\
02 \text { pacientes em UTI, } \\
\text { permitindo a redução } \\
\text { experimental, } \\
\text { segurança da PEEP, de } \\
25 \text { para } 5 \mathrm{cmH} 2 \mathrm{O}\end{array}$ \\
\hline \multicolumn{5}{|c|}{$\begin{array}{l}\text { Limitações: Revisão narrativa pouco abrangente. Sem comparação direta entre as alternativas para } \\
\text { monitorização pulmonar abordadas }\end{array}$} \\
\hline $\begin{array}{l}\text { O. Moerer, G. } \\
\text { Hahn e M. } \\
\text { Quintel, } 2011\end{array}$ & $\begin{array}{lr}\text { Revisão de } & \text { literatura. } \\
\text { Pacientes } & \text { com } \\
\text { insuficiência respiratória } \\
\text { aguda submetidos a } \\
\text { ventilação r mecânica } \\
\text { invasiva }\end{array}$ & $\begin{array}{l}\text { Uso TIE para } \\
\text { monitorização } \\
\text { pulmonar }\end{array}$ & $\begin{array}{l}\text { Estresse e } \\
\text { tensão do } \\
\text { parênquima } \\
\text { pulmonar }\end{array}$ & $\begin{array}{l}\text { Parece haver evidências } \\
\text { que suportam o uso de } \\
\text { EIT como medida de } \\
\text { cabeceira, a fim de } \\
\text { prevenir a indução de } \\
\text { lesão pulmonar }\end{array}$ \\
\hline \multicolumn{5}{|c|}{$\begin{array}{l}\text { Limitações: Revisão narrativa pouco abrangente. Sem comparação direta entre as alternativas para } \\
\text { monitorização pulmonar abordadas }\end{array}$} \\
\hline $\begin{array}{l}\text { P. Theerawit et } \\
\text { al, } 2017\end{array}$ & $\begin{array}{lr}\text { Revisão de } & \text { literatura. } \\
\text { Pacientes } & \text { com } \\
\text { insuficiência } & \text { respiratória } \\
\text { aguda } & \end{array}$ & $\begin{array}{l}\text { Diferentes } \\
\text { métodos para } \\
\text { monitoração } \\
\text { respiratória em } \\
\text { UTI }\end{array}$ & $\begin{array}{l}\text { Otimização } \\
\text { da ventilação } \\
\text { mecânica }\end{array}$ & $\begin{array}{l}\text { Os sinais de pulmão na } \\
\text { TIE são susceptíveis a } \\
\text { interferências por várias } \\
\text { fontes incluindo a } \\
\text { ventilação de alta } \\
\text { frequência } \\
\text { monitorização } \\
\text { hemodinâmica invasiva }\end{array}$ \\
\hline \multicolumn{5}{|c|}{$\begin{array}{l}\text { Limitações: Revisão narrativa pouco abrangente. Sem comparação direta entre as alternativas para } \\
\text { monitorização pulmonar abordadas }\end{array}$} \\
\hline $\begin{array}{l}\text { L. Yun et al, } \\
2016\end{array}$ & $\begin{array}{l}\text { Observacional } \\
\text { prospectivo. } 20 \text { pacientes } \\
\text { maiores de } 18 \text { anos com } \\
\text { síndrome de dificuldade } \\
\text { respiratória }\end{array}$ & $\begin{array}{l}\text { Uso do TIE para } \\
\text { avaliar } \\
\text { Manobras de } \\
\text { recrutamento } \\
\text { alveolar }\end{array}$ & $\begin{array}{l}\text { Otimização } \\
\text { da relação } \\
\text { ventilação- } \\
\text { perfusão }\end{array}$ & $\begin{array}{l}\text { O TIE demonstrou } \\
\text { diferença significativa no } \\
\text { índice relativo ventilação- } \\
\text { perfusão foi encontrado } \\
\text { entre os pacientes com } \\
\text { notável reabertura } \\
\text { pulmonar quando } \\
\text { comprado ao grupo com } \\
\text { insuficiente reabertura } \\
\text { após manobra de } \\
\text { recrutamento alveolar ( } P \\
<0,01) \text { o que foi } \\
\text { confirmado } \\
\text { gasometria }\end{array}$ \\
\hline \multicolumn{5}{|c|}{ Limitacões: Não teve comparador para o TIE } \\
\hline $\begin{array}{l}\text { R. Rosa et al, } \\
2015\end{array}$ & $\begin{array}{l}\text { Estudo de Caso. Paciente } \\
\text { de } 63 \text { anos do sexo } \\
\text { masculino com síndrome } \\
\text { do desconforto } \\
\text { respiratório agudo }\end{array}$ & $\begin{array}{l}\text { Uso TIE } \\
\text { para auxiliar às } \\
\text { manobras de } \\
\text { recrutamento } \\
\text { alveolar }\end{array}$ & $\begin{array}{l}\text { Melhora da } \\
\text { hipoxemia }\end{array}$ & $\begin{array}{l}\text { A TIE identificou, após } \\
\text { manobras de } \\
\text { recrutamento, melhora na } \\
\text { distribuição da ventilação } \\
\text { pulmonar, evidenciando } \\
\text { maior ventilação em } \\
\text { áreas previamente } \\
\text { colapsadas possibilitando } \\
\text { a manutenção dos } \\
\text { parâmetros ventilatórios } \\
\text { com volume corrente de } \\
6 \mathrm{~mL} / \mathrm{kg} \text { predito de peso e } \\
\mathrm{PEEP} \text { de } 16 \mathrm{~cm} \text { H2O, com } \\
\text { pressão de platô de } \\
26 \mathrm{cmH} 2 \mathrm{O} \text { e a relação } \\
\mathrm{PaO} / \mathrm{FiO} 2 \text { era de }\end{array}$ \\
\hline
\end{tabular}

Rev. Saúde Digital Tec. Educ., Fortaleza, CE, v. 5, n.1, p.16-36, jan./abr. 2020. 


\begin{tabular}{|c|c|c|c|c|}
\hline & & & & $226 \mathrm{mmHg}$ \\
\hline \multicolumn{5}{|c|}{ Limitações: Não teve comparador para o TIE } \\
\hline $\begin{array}{l}\text { T. Kotani et al, } \\
2015 .\end{array}$ & $\begin{array}{l}\text { Estudo de Caso. Paciente } \\
\text { de } 77 \text { anos, do sexo } \\
\text { feminino, com cor } \\
\text { pulmonale agudo e } \\
\text { síndrome de dificuldade } \\
\text { respiratória aguda grave }\end{array}$ & $\begin{array}{l}\text { Uso do TIE para } \\
\text { monitorar } \\
\text { manobras e } \\
\text { recrutamento } \\
\text { alveolar }\end{array}$ & $\begin{array}{l}\text { Melhora da } \\
\text { hipoxemia }\end{array}$ & $\begin{array}{l}\text { O recrutamento de } \\
\text { pulmões monitorado pelo } \\
\text { EIT foi paralelo à } \\
\text { melhoria de } \mathrm{PaO} / \mathrm{FIO} 2 \\
\text { de } 123 \text { a } 239 \mathrm{mmHg} \text {. } \\
\text { paciente foi desabituado } \\
\text { da ventilação mecânica } \\
\text { depois de } 32 \text { dias }\end{array}$ \\
\hline \multicolumn{5}{|c|}{ Limitações: Não teve comparador para o TIE } \\
\hline $\begin{array}{l}\text { M. } \\
\text { Stankiewicz- } \\
\text { Rudnicki, T. } \\
\text { Gaszyński, W. } \\
\text { Gaszyński, } \\
2015\end{array}$ & $\begin{array}{l}\text { Revisão da literatura. } \\
\text { Pacientes com síndrome } \\
\text { de dificuldade respiratória } \\
\text { aguda }\end{array}$ & $\begin{array}{l}\text { Uso do TIE para } \\
\text { otimização da } \\
\text { ventilação } \\
\text { mecânica }\end{array}$ & $\begin{array}{l}\text { Distribuição } \\
\text { de ventilação } \\
\text { regional }\end{array}$ & 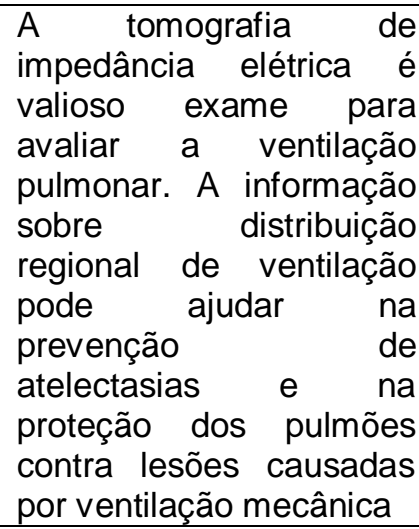 \\
\hline \multicolumn{5}{|c|}{ Limitações: Revisão narrativa e pouco abrangente. Não teve comprador para o TIE } \\
\hline $\begin{array}{l}\text { B. Walsh e C. } \\
\text { Smallwood, } \\
2016\end{array}$ & Revisão de literatura. & $\begin{array}{l}\text { Uso no TIE para } \\
\text { otimização da } \\
\text { ventilação } \\
\text { mecânica }\end{array}$ & $\begin{array}{l}\text { Distribuição } \\
\text { de ventilação } \\
\text { regional }\end{array}$ & $\begin{array}{l}\text { O TIE mostrou-se útil na } \\
\text { detecção de } \\
\text { pneumotórax, } \\
\text { quantificação de edema } \\
\text { pulmonar e comparação } \\
\text { da distribuição de de } \\
\text { ventilação } \\
\text { diferentes modos de } \\
\text { ventilação e pode } \\
\text { oferecer um indivíduo } \\
\text { superior titulação de } \\
\text { PEEP e outros } \\
\text { parâmetros do ventilador } \\
\text { comparados con com } \\
\text { abordagens existentes }\end{array}$ \\
\hline \multicolumn{5}{|c|}{ Limitações: Revisão narrativa e pouco abrangente. Não teve comprador para o TIE } \\
\hline $\begin{array}{l}\text { J. Kobylianskii } \\
\text { et al, } 2016\end{array}$ & $\begin{array}{lr}\text { Revisão } & \text { Sistemática. } \\
\text { Pacientes } & \text { adultos } \\
\text { ventilados } & \\
\text { mecanicamente. }\end{array}$ & $\begin{array}{l}\text { Uso do TIE em } \\
\text { pacientes adultos } \\
\text { submetidos a } \\
\text { mecânica } \\
\text { Ventilação }\end{array}$ & $\begin{array}{l}\text { Não foi } \\
\text { claramente } \\
\text { definido }\end{array}$ & $\begin{array}{l}\text { Dos } 67 \text { estudos incluídos, } \\
35 \text { tiveram o objetivo de } \\
\text { validar medidas de TIE, } \\
\text { 32, avaliar o uso do TIE } \\
\text { para monitorar a resposta } \\
\text { a intervenções } \\
\text { terapêuticas em adultos. } \\
\text { O TIE demonstrando-se } \\
\text { útil na medição da } \\
\text { mecânica respiratória } \\
\text { regional. Parece existir } \\
\text { evidências consideráveis } \\
\text { quanto à confiabilidade e } \\
\text { reprodutibilidade do uso } \\
\text { do TIE, e sua utilidade no } \\
\text { monitoramento }\end{array}$ \\
\hline
\end{tabular}

Rev. Saúde Digital Tec. Educ., Fortaleza, CE, v. 5, n.1, p.16-36, jan./abr. 2020. 


\begin{tabular}{|c|c|c|c|c|}
\hline & & & & $\begin{array}{l}\text { facilitação de melhorias } \\
\text { da ventilação mecânica. } \\
\text { Permanece a questão de } \\
\text { saber se é clinicamente } \\
\text { relevante na melhoria } \\
\text { dos cuidados ao paciente }\end{array}$ \\
\hline \multicolumn{5}{|c|}{$\begin{array}{l}\text { Limitações: A questão de pesquisa não era clara. Incluiu somente estudos no idioma inglês. Alto risco de } \\
\text { viés (ROBIS) }\end{array}$} \\
\hline $\begin{array}{l}\text { A. Romero, B. } \\
\text { Alonso, I. } \\
\text { Latorre, J. } \\
\text { García, } 2015\end{array}$ & $\begin{array}{l}\text { Estudo de Caso. Paciente } \\
\text { adulto do sexo masculino } \\
\text { submetido a transplante } \\
\text { uni pulmonar esquerdo } \\
\text { devido a DPOC do } \\
\text { enfisema. }\end{array}$ & $\begin{array}{l}\text { Uso do TIE em } \\
\text { pacientes adultos } \\
\text { submetidos a } \\
\text { mecânica } \\
\text { Ventilação }\end{array}$ & $\begin{array}{l}\text { Distribuição } \\
\text { de ventilação } \\
\text { regional }\end{array}$ & $\begin{array}{l}\text { O TIE provou ser uma } \\
\text { técnica, à beira do leito, } \\
\text { que pode monitorar as } \\
\text { mudanças no volume } \\
\text { pulmonar durante a a } \\
\text { ventilação mecânica. } \\
\text { Nesse caso, foi possível } \\
\text { com o TIE identificar o } \\
\text { ganho de volume } \\
\text { pulmonar após manobra } \\
\text { de recrutamento alveolar }\end{array}$ \\
\hline \multicolumn{5}{|c|}{ Limitações: Não teve comparador para o TIE } \\
\hline $\begin{array}{l}\text { J. Karsten et } \\
\text { al, } 2015 .\end{array}$ & $\begin{array}{l}\text { Observacional } \\
\text { prospectivo. } 20 \text { pacientes } \\
\text { adultos em PO de cirurgia } \\
\text { cardíaca com síndrome } \\
\text { da dificuldade respiratória } \\
\text { aguda }\end{array}$ & $\begin{array}{l}\text { Uso do TIE em } \\
\text { pacientes adultos } \\
\text { submetidos a } \\
\text { mecânica } \\
\text { Ventilação }\end{array}$ & $\begin{array}{l}\text { Distribuição } \\
\text { de ventilação } \\
\text { regional// } \\
\text { Conformidade } \\
\text { dinâmica } \\
\text { global(CRS) }\end{array}$ & $\begin{array}{l}\text { O teste de PEEP } \\
\text { descendente durou } \\
\text { menos de } 10 \text { min. O TIEI } \\
\text { revelou que a CRS } \\
\text { aumentou após manobra } \\
\text { de recrutamento alveolar } \\
\text { (PEEP máximo de } 8 \\
\text { cmH2O). O "melhor" } \\
\text { PEEP individual por CRS } \\
\text { mostrou } \\
\text { significativamente valores } \\
\text { mais baixos do que o } \\
\text { "melhor" PEEP por } \\
\text { distribuição de volume } \\
\text { medido com índices EIT }\end{array}$ \\
\hline \multicolumn{5}{|c|}{ Limitações: Não teve comparador para o TIE } \\
\hline $\begin{array}{l}\text { T. Luecke, } F . \\
\text { Corradi, } \\
\text { Pelosib, } 2012\end{array}$ & $\begin{array}{l}\text { Revisão de literatura. } \\
\text { Pacientes com síndrome } \\
\text { de dificuldade respiratória } \\
\text { aguda }\end{array}$ & $\begin{array}{l}\text { Uso do TIE em } \\
\text { pacientes adultos } \\
\text { submetidos a } \\
\text { mecânica } \\
\text { Ventilação }\end{array}$ & $\begin{array}{l}\text { Recrutamento } \\
\text { alveolar e } \\
\text { distribuição } \\
\text { de ventilação } \\
\text { regional }\end{array}$ & $\begin{array}{l}\text { Para monitorar } \\
\text { recrutamento e a } \\
\text { hiperinflação do tecido } \\
\text { pulmonar em diferentes } \\
\text { níveis de pressão de } \\
\text { inflação, o tomógrafo de } \\
\text { impedância elétrica } \\
\text { emerge como ferramenta } \\
\text { não invasiva e sem } \\
\text { radiação valiosa que } \\
\text { pode ser usada em } \\
\text { conjunto com outros } \\
\text { parâmetros globais para } \\
\text { adquirir informações } \\
\text { adicionais } \\
\text { recrutamento sobre } \\
\text { distribuição de ventilação }\end{array}$ \\
\hline \multicolumn{5}{|c|}{ Limitações: Revisão narrativa e pouco abrangente. Não comparou diretamente o TIE } \\
\hline $\begin{array}{l}\text { F. A Lopes et } \\
\text { al, } 2016\end{array}$ & $\begin{array}{l}\text { Estudo de caso. Mulher } \\
\text { de } 65 \text { anos de idade com } \\
\text { história de cirurgia }\end{array}$ & $\begin{array}{lr}\text { Uso do } & \text { TIE } \\
\text { (Timpel } & \text { Enlight } \\
\text { 1800) } & \text { no }\end{array}$ & $\begin{array}{l}\text { Tempo para a } \\
\text { desabituação } \\
\text { do ventilador }\end{array}$ & $\begin{array}{l}\mathrm{O} \text { TIE ajudou na } \\
\text { interpretação de falhas } \\
\text { na retirada do suporte }\end{array}$ \\
\hline
\end{tabular}

Rev. Saúde Digital Tec. Educ., Fortaleza, CE, v. 5, n.1, p.16-36, jan./abr. 2020.

ISSN: 2525-9563 


\begin{tabular}{|l|l|l|l|l|}
\hline & $\begin{array}{l}\text { Substituição da valva } \\
\text { mitral por uma bioprótese } \\
14 \text { anos antes. }\end{array}$ & $\begin{array}{l}\text { desmame } \\
\text { ventilatório }\end{array}$ & & $\begin{array}{l}\text { ventilatório, possibilitando } \\
\text { diagnóstico de } \\
\text { pendelluft, sem expor o } \\
\text { paciente às medidas } \\
\text { invasivas de outras } \\
\text { formas de monitoramento }\end{array}$ \\
\hline Limitações: Único braço, sem comparador & & \\
\hline
\end{tabular}

Fonte: os autores

Rev. Saúde Digital Tec. Educ., Fortaleza, CE, v. 5, n.1, p.16-36, jan./abr. 2020. 


\section{Como citar este artigo}

Silva RCL da; Peregrino AAF; Machado DA; Silva CRL da; Oliveira CR de; Marta CB. Efetividade da tomografia de impedância elétrica torácica na monitorização de pacientes em ventilação mecânica. Revista de Saúde Digital e Tecnologias Educacionais. [online], volume 5, n. 1. Editor responsável: Luiz Roberto de Oliveira. Fortaleza, mês e ano, p. 1636. Disponível em: http://periodicos.ufc.br/resdite/index. Acesso em "dia/mês/ano".

Data de recebimento do artigo: 08/09/2019

Data de aprovação do artigo: 11/02/2020

Data de publicação: 17/04/2020

Rev. Saúde Digital Tec. Educ., Fortaleza, CE, v. 5, n.1, p.16-36, jan./abr. 2020. 